\title{
Search of visually similar microscopic rock images
}

\author{
Magdalena Ladniak • Mariusz Młynarczuk
}

Received: 24 June 2014 / Accepted: 29 October 2014 / Published online: 27 November 2014

(C) The Author(s) 2014. This article is published with open access at Springerlink.com

\begin{abstract}
The present paper discusses the essential tenets of the method whose purpose is to enable effective search of images of given rock in multimedia databases. The search is based exclusively on an image request, to which the system's response is a set of images presenting visually similar rocks. The images that constitute the basis of the discussed research had been registered with an optical microscope. The collection of images that were used in the process of performing measurements encompassed 5700 digital images presenting 19 rock types. The proposed method is based on the application of image analysis and artificial intelligence concepts. The very process of inference, in turn, makes use of the methods of data classification and grouping. In the paper, the authors demonstrate that these may turn out to be effective mathematical methods, successfully applied to the problem of image search, performed with imagings presenting rock textures. The discussed system concept, based on a feature space defined by the authors, successfully matches up images with the reference standard. The effectiveness rate of that process is very high (very often, it is $100 \%$ ). Failed classifications concern only the images which differ visually-in a considerable way-from the rest of the images within a given group. The proposed system concept is to facilitate the decisionmaking process involved in determining the similarity of investigated objects. In the opinion of the authors, it meets
\end{abstract}

M. Ładniak $(\triangle) \cdot$ M. Młynarczuk

Faculty of Geology, Geophysics and Environmental Protection, AGH University of Science and Technology, al. Mickiewicza 30, 30-059, Cracow, Poland

e-mail:mladniak@agh.edu.pl

M. Młynarczuk

e-mail:mlynar@agh.edu.pl the requirements - and, as such, can be applied to the problem of searching for images in databases, searching discs in order to find images of given rocks and automatic information gain on the basis of video sequences, e.g., in order to find frames presenting particular rock structures.

Keywords Search by image - Image exploration . Artificial intelligence $\cdot$ Cluster analysis · Data analysis . Image processing

\section{Introduction}

In recent years, obtaining, storing, and sharing multimedia data have been influenced by the dynamic development of information technology. It is no longer the case that multimedia data is connected with a given PC or storage medium - on the contrary, it is widely available as databases, stored on network servers and accessed simultaneously by a number of users (Fig. 1). Such a tendency necessitates optimizing the way in which the data in question is organized, analyzed, and interpreted. In particular, this concerns visual data (such as digital photos or videos). It is a dynamically developing issue in medicine and the broader bioinformatics $[28,29]$. Such data is successfully explored by means of methods of digital image processing (e.g., the ones that facilitate stereological analysis) [22] and [12] and methods involving artificial intelligence (such as teaching neural networks) [27]. The combination of these methods results in obtaining highly specialized algorithms of image content analysis. Such issues are also investigated in relation to geological and mining data $[24,25]$ and the results reported by researchers seem to be very promising. However, it needs to be remembered that obtaining highquality analysis results—-such as those presented by the 
Fig. 1 Sharing research resources among multiple scientific and industrial units

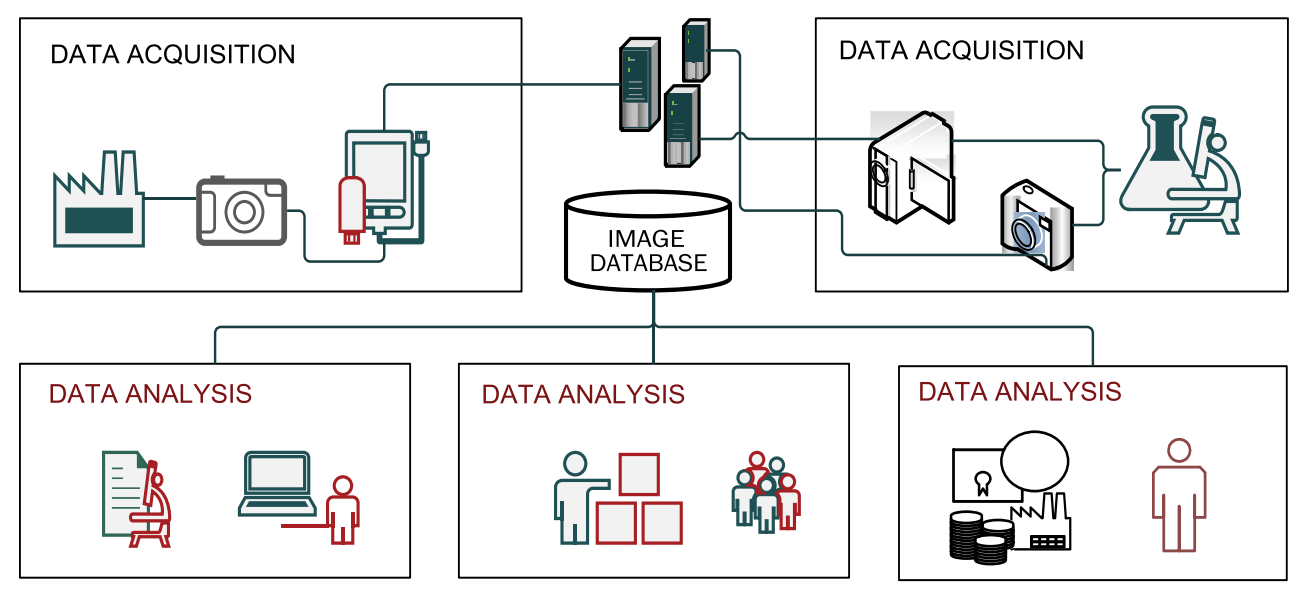

authors of well-known scientific publications [3] and [16] and [19]—is connected with using sophisticated learning data sets, due to which proposed algorithms make decisions concerning the analysis of investigated objects.

Such an approach, though very efficient, cannot be adopted when exploring a database that was not previously described, i.e., a database about which the algorithm has no a priori knowledge, neither in the form of text descriptions, nor in the form of previously defined learning sequences. An example can be the problem of searching for visually similar rock structure images. The key to such a search is one image, solely. Thus, the authors propose to use nonmodel methods of data exploration in order to construct an algorithm facilitating the process of determining the visual similarity between rock structure imagings.

\section{Image search-a description}

Usually, the technological progress in mining and geology requires thorough reconnaissance of rocks and a rock massif. This often involves the obligatory determination of parameters of the observed rock structures. Here, image analysis [9] and [14] proves an effective method, which eventually leaves researchers with constantly increasing databases containing digital multimedia materials. The everincreasing amount of data in such data collections, as well as consolidating collections from various independent research teams at big scientific and industrial consortiums (Fig. 1), make the processes of exploring databases and searching for the desired images extremely difficult [17].

In relevant sources, two approaches to image search (Fig. 2)-based on their content-prevail (visual image retrival (VIR) systems). The first approach focuses on the usage of a text, viewed as the main information carrier. Both in the search query and in the search results, textual descriptions of images saved in the form of metadata are used (first-generation VIR systems, text-based image retrieval $(T B I R))$. The other approach is based on image content similarity (second-generation content-based VIR (CBVIR) systems) $[2,13,26]$. Searching performed just on the basis of textual metadata related to a given image (TBIR)—such as size, written content description, etc.- - seems to be inadequate in the task of searching and identifying rock textures successfully. It is true that textual data, describing image content, can be searched through easily (text mining and

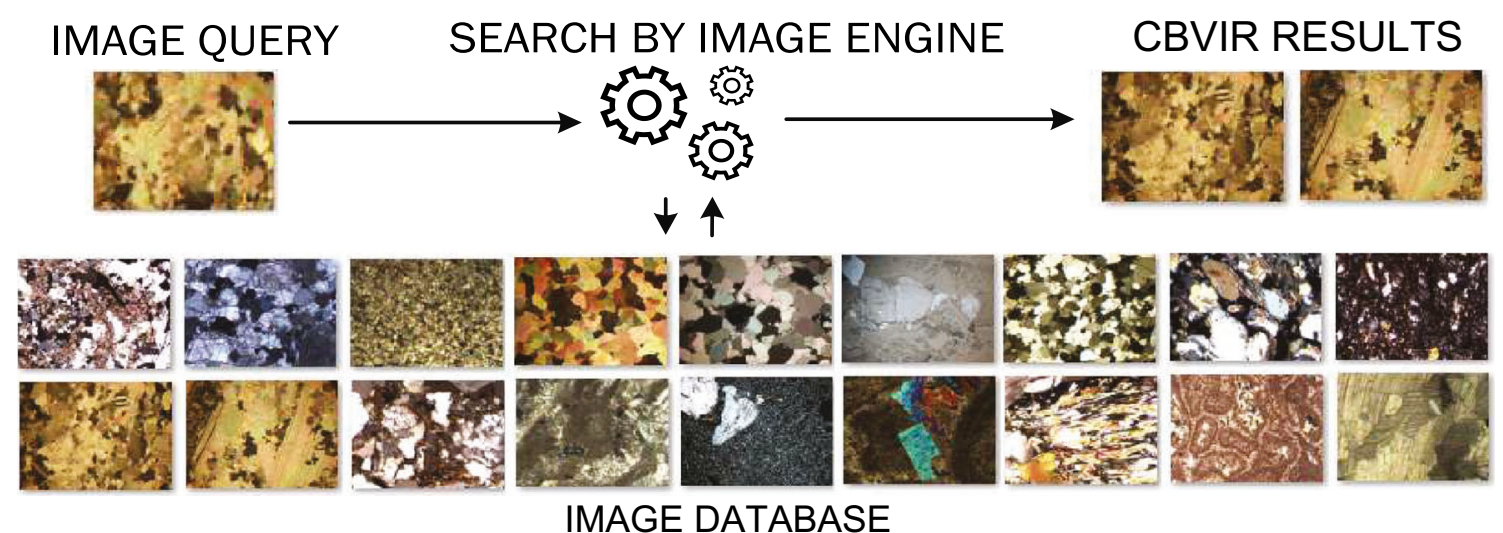

Fig. 2 The concept of search of visually similar rock structure imagings 
questions related to it have been thoroughly researched) [7]—still, using this type of data involves providing a written description for each and every image included in a database. This comes out highly impractical in the case of really large image databases, often generated in an automatic manner (e.g., by means of a microscope). Therefore, the problem of image search performed on the basis of an automatic analysis of their content (CBVIR) has been tackled. Such a strategy reflects, to a considerable degree, the way the human mind performs such tasks $[1,8]$. In the case of images presenting rock textures, such a comparison involves mainly observing variability in the image color and texture. The authors propose their own concept of an image search algorithm to be used in order to search for visually similar images within a given, analyzed data base. The user chooses the search key (query) in the form of an image. What needs to be stressed is that the user does not have to possess expert knowledge to precisely determine search criteria. The only piece of information that they can supply (other than choosing the image key) is the number of similar images in k-nearest neighbours $(\mathrm{kNN})$ algorithm or number of groups in others methods, that they expect to obtain as a result of the search process. The system, having analyzed the data, makes a decision as to the best visual match to the search key. By the image search method, we shall understand a set of operations making it possible to compare images within the analyzed set (Fig. 3). The authors propose to extract these characteristics which-like human mind-are responsible for differentiating images within a searched database, in a possibly unambiguous way. This involves the methods for processing digital images. A newly created set of characteristics, in a numerical form, thus becomes a set of input data for artificial intelligence methods, which draw inferences regarding their similarity. The obtained collections of images are the search results.

\section{Methodology of determining image similarity}

The research material was microscope images of thin sections of 19 rock types. The analyzed rocks came from the southern regions of the Polish (Carpathian and Sudeten) Mountains. From these rocks, thin sections were made. The photos were taken with a polarized light optical microscope, at the optimum illumination and magnification which was the same for all the photos registered for a given type of rock. For each rock type, 300 photos were taken (Figs. 4 and 5). In all, the collection of photos subjected to further analysis encompassed 5700 digital images. It is essential to observe that the analyzed rocks did not demonstrate visual homogeneity within one particular rock type. Practically speaking, this means that rocks constituting one

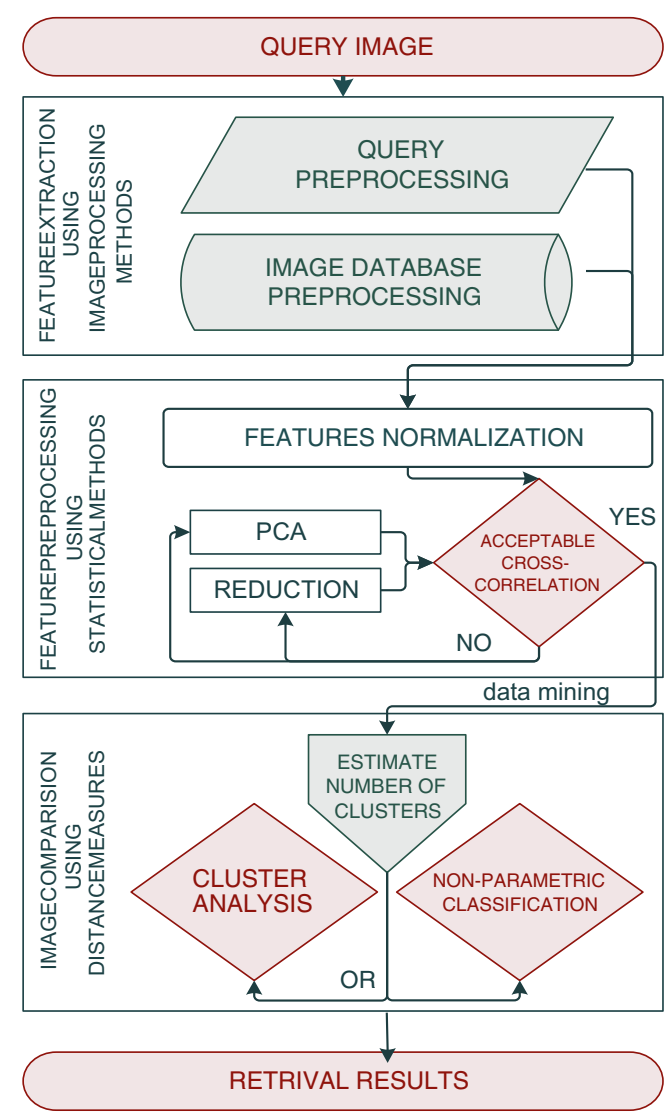

Fig. 3 Data flow in the image search algorithm

petrographic group revealed a tendency, visually, towards falling into separate, different groups (Fig. 5).

\subsection{Determination of the characteristics identifying photographs}

In the literature of the subject, two approaches to search by image content are dominant. One involves searching performed on the basis of general image characteristics, such as color or texture. The other approach, called a semantic one, involves representing relationships between objects in each photo. The methodology adopted in the present paper uses the main ideas of both approaches. Most importantly, the analyzed material revealed differences as far as color and texture were concerned. Texture represents such characteristics as the arrangement of heterogenous areas within an image, i.e., the way in which minerals within a body of rock were ordered spatially. The authors used this as a basis for determining the parameters of content differentiation (Table 1). Due to the particular nature of the research, the stereological parameters of materials were not used, as their extraction would necessitate adopting different segmentation algorithms [20] for different rock types. Such an approach rules out their universal usability in relation to the 


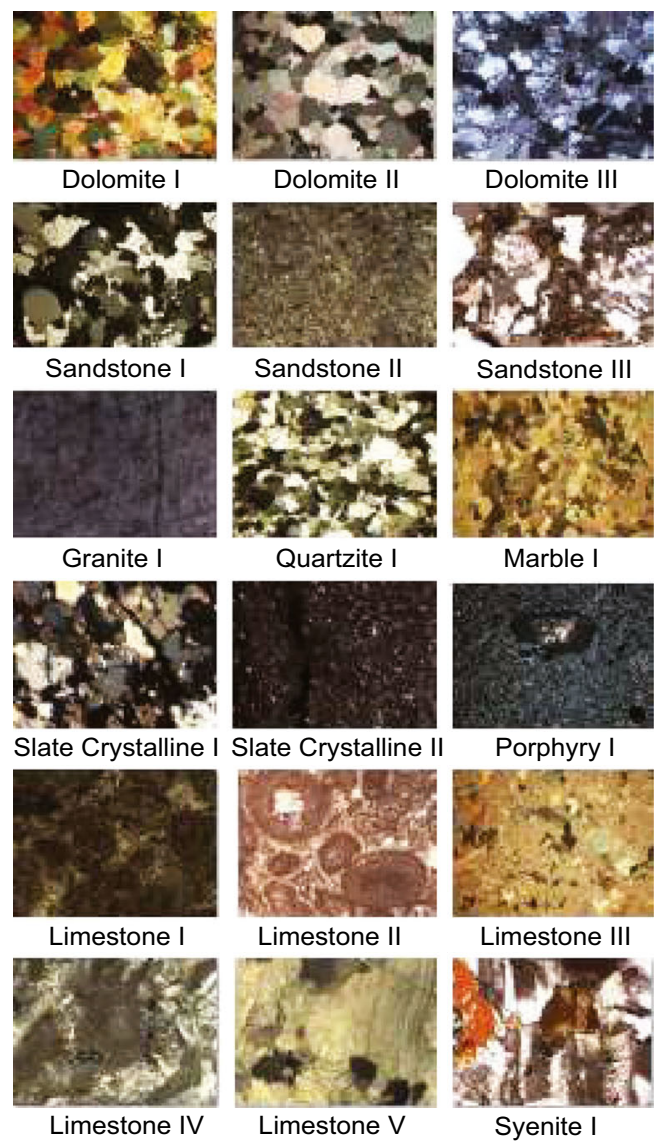

Fig. 4 Representatives of selected thematic categories, i.e., petrographic types of rocks

construction of the proposed system. Thus, a unified algorithm was proposed for all rock types, to be used for the purpose of determining the number of objects included in a given image. All the analyses were carried out on the basis of the RGB and CIELAB color model. The parameters were determined by means of methods of digital image processing.

For the analyzed data, the usefulness of other parameters was also investigated. The parameters describing the fraction of universal simple colors and their modifications (red, green, blue, yellow, magenta, cyan) within each

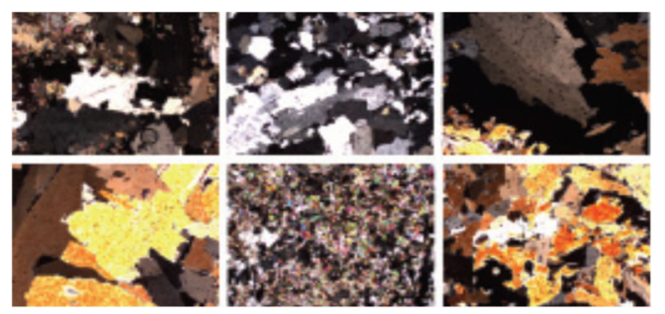

Fig. 5 Visual diversification within one petrographic groupanhydrite picture proved useful. They were also used in the research (six parameters). Unfortunately, the static parameterssuch as those describing the distribution of pixel values within an image-turned out to be less helpful. A sample parameter was the value of the concentration of the distribution of particular gray levels of color channels and the luminance channels. Theoretically, this testifies to the uneven distribution of the values of a characteristic among the elements of a sample. Another characteristic was the value of the asymmetry of the distribution of the values of particular gray levels of color channels and the luminance channels, which supplied information about the distribution symmetry or lack of it. It is a good separating parameter for a small set of diversified rock structures, but, for the set encompassing 19 rock types, it is an insufficient differentiating parameter.

As a result of the research, 41 basic parameters were selected. They were subsequently subjected to further analyses so that similarity between images could be evaluated. To this end, the authors proposed performing image projection within a previously defined space of features. This space may be treated as an n-dimensional Euclidean space, where further calculations can be performed. In this context, an object is constituted by an individual image, projected within a Euclidean space in the form of a feature vector, whose components are the values determining the measures of selected descriptive parameters of an image [18]. The Euclidean distance between these objects was established to be the measure of differences between the objects (images). The process of obtaining the values of the descriptive features of each image resulted in the creation of a set of data (the description of a feature space) of the size $P \times L$, where $P=$ the number of objects in the analyzed set of data, $L=$ the number of parameters describing each of the elements. Thus, obtained data had to be processed statistically before any inferences could be drawn, as the attribute of the numerically highest value tended to dominate over the others. This makes it impossible to project the issue to the space of features described above in an expert way. The solution was to normalize the values of the parameters, which involved scaling the interval to which they belong to the interval $[0,1]$.

\subsubsection{Verification of feature representativeness}

The set of data obtained in the course of previous stages reveals a considerable correlation between the measure of particular features in the representations of particular color channels of the RGB model (ca. 46-99 \%), and a less considerable one in the CIELAB model (ca. 20-50\%). The existence of strongly correlated values increases the influence of a given feature on the other ones in the process of analyzing particular components of 
Table 1 Parameters of image description and the procedures for obtaining them

\begin{tabular}{|c|c|c|c|}
\hline No. & Name & Meaning & Procedure \\
\hline 1 & Image volume & Averaged coloring & $\begin{array}{l}\text { Determine the mean value of pixel gray levels of the image for particular } \\
\text { color channels and the luminance channel, ( } 7 \text { parameters })\end{array}$ \\
\hline 2 & $\begin{array}{l}\text { Morphological gradient } \\
\text { volume }\end{array}$ & $\begin{array}{l}\text { Frequency of boundaries } \\
\text { and image noise }\end{array}$ & $\begin{array}{l}\text { Determine the mean value of all pixel gray levels of the morphological } \\
\text { gradient image for particular color channels and the luminance } \\
\text { channel, ( } 7 \text { parameters })\end{array}$ \\
\hline 3 & $\begin{array}{l}\text { Color variability of } \\
\text { image segments }\end{array}$ & $\begin{array}{l}\text { Coefficient of image } \\
\text { colors variability }\end{array}$ & $\begin{array}{l}\text { Divide the image into } N \times M \text { (here } N=M=10 \text { ) blocks; for each block, } \\
\text { determine the sum of pixel values for particular color channels and } \\
\text { the luminance channel; determine the variability coefficient for the } \\
\text { created } N \times M \text { matrix (the variability coefficient is defined as the } \\
\text { standard deviation of the sample divided by its arithmetic } \\
\text { mean), ( } 7 \text { parameters) }\end{array}$ \\
\hline 4 & Image semivariogram & $\begin{array}{l}\text { Variability of color in } \\
\text { the neighborhood of } h\end{array}$ & $\begin{array}{l}\text { Determine the value of the semivariogram function for the established } \\
\text { range of distances } h \text { (here } h=120) \text {, calculated for particular color } \\
\text { channels and the luminance channel, ( } 7 \text { parameters })\end{array}$ \\
\hline 5 & $\begin{array}{l}\text { Number of diversified } \\
\text { image objects }\end{array}$ & Texture variability & $\begin{array}{l}\text { Filtrate the morphological gradient image with a median filter using } \\
\text { a } n \times n \text { mask (here } n=5 \text { ); perform the watershed operation upon the } \\
\text { image complement; calculate the number of detected segments for } \\
\text { particular color channels and the luminance channel, ( } 7 \text { parameters) }\end{array}$ \\
\hline
\end{tabular}

descriptive vectors. The authors suggest their explicit elimination by selecting - for the purpose of further inferencethese features whose Pearson's correlation coefficient reaches the minimum. Another way of eliminating the redundant features is by adopting the methods of the principal component analysis (PCA). This is done by such a rotation of a coordinate system (a set of data composed of $P$ observations, each of which encompasses $L$ variables, can be interpreted as a $P$ point cloud within an $L$-dimensional space) that aims at maximizing, in the first place, the variance of the first coordinate, then the variance of the second coordinate, etc. [5, 21]. This, eventually, results in an opportunity to reduce the size of the data matrix by eliminating statistically the least relevant (i.e., already transformed) vectors. This method does not provide us directly with any information as to the eliminated parameters.

\subsection{Methods of obtaining knowledge about image clusters}

A fully automatic analysis of rock structures is a difficult process due to the lack of regularity in the visual description of the research material. A relatively high effectiveness in the process of the automatic recognition of rock structures is revealed by the methods of machine learning with a precisely selected and prepared learning sequence which is a fair representation of the analyzed population. The authors of research [3], [19], and [25] achieve high efficiency in that respect, often reaching $98 \%$. Unfortunately, such an approach seems impossible to be adopted whenever there is a need to explore a database of images that have not been described in any way. In such cases, it becomes impossible to isolate a representative learning sequence, as no detailed information about it is available. Thus, the authors propose using non-model methods that combine data exploration and machine learning from data. These methods have been widely discussed in various sources, therefore, the authors shall abstain from a theoretical introduction and refer to the already existing publications instead.

\subsubsection{Methods of cluster analysis}

The first possibility is using the methods of cluster analysis as a method of data self-organization, i.e., clustering. This method groups elements into relatively homogenous classes. The search key is, in this case, integrated with a test set, the process of grouping is performed, and then the data of the friendly group is returned, on the basis of the metric of distance in the Euclidean coordinate set. The application of these methods necessitates the estimation of a number of groups that can be arrived at-by giving this number a priori or estimating it with some automatic methods, e.g., via gap statistic $[10,11,30]$. Four algorithms of data clustering were tested: the k-means algorithm [23], the $k$-medoids algorithm [15], the minimum entropy clustering (MEC) algorithm [4], and the method of fuzzy cluster analysis-the c-means algorithm [31]. Below, the results of the evaluation carried out for different image group sizes were presented. 
Table 2 The compatibility of image search results with their geological origin, presented for various parameter variations (the $k$-means data grouping method) $(\%)$

\begin{tabular}{|c|c|c|c|c|c|c|c|c|c|}
\hline Number of groups & 19 & 30 & 40 & 50 & 60 & 70 & 80 & 90 & 100 \\
\hline CIELAB & 80.23 & 89.01 & 89.44 & 90.12 & 92.91 & 93.71 & 94.06 & 95.46 & 94.61 \\
\hline RGB & 76.24 & 77.53 & 84.34 & 84.28 & 86.73 & 86.28 & 89.14 & 90.10 & 90.55 \\
\hline CIELAB, PCA = 3 & 68.26 & 74.79 & 77.78 & 77.82 & 80.31 & 79.78 & 81.08 & 81.76 & 79.37 \\
\hline CIELAB, PCA = 5 & 73.33 & 81.05 & 84.47 & 85.29 & 85.51 & 86.47 & 89.47 & 87.98 & 91.03 \\
\hline CIELAB, PCA $=7$ & 72.38 & 82.80 & 86.94 & 88.18 & 90.76 & 90.24 & 91.24 & 92.02 & 92.51 \\
\hline
\end{tabular}

\subsubsection{Method of search key classification based on the analysis of k-nearest neighbors}

Another way of obtaining information on the similarity of objects (images) is using classification methods based on the distance analysis ( $k$-nearest neighbors, $k N N$ ). In the context of image search, this method-in its tenets-seems to satisfy the system needs. The user requests that the system finds $k$-most similar images in the whole database available. The parameter $k$ - determining the number of nearest neighbors taken into account during the classification process-is very essential with regards to the effectiveness of the solutions and the complexity of calculations [6].

\section{Evaluating the accuracy of searching rock structures by image}

The automatic evaluation of the similarity between images presenting rock structures is a difficult task, as it is very often the case that geological objects from one category have different structures, which can be assessed visually (Fig. 5). To eliminate the risk of passing a one-sided judgement, the authors presented the results of the system's performance in the light of their compatibility with the actual rock types and their visual matching. The accuracy of the system's performance was expressed as the percentage of correctly identified objects (the mean percentage of correctly classified objects for 100 repeated applications of each method). The results were presented in the form of a table so that the reader could assess them objectively. Additionally, sample extreme visual search results (in terms of effectiveness) were given.

\subsection{Evaluating the elimination of redundant parameters}

The presented basic set of features reveals a considerable correlation of its own values. This increases the influence of one feature on the other ones. The authors present the comparison of the elimination of the data that contribute the least to effective clustering performed with artificial intelligence methods. The comparison concerned the results of selecting data with the lowest (the CIELAB model) and the highest (the RGB model) interclass correlation. The results of search accuracy for various numbers of similar image groups were also presented. Additionally, the authors present the effects resulting from the usage of the PCA algorithm with regards to obtaining the most accurate end results (the CIELAB model). The results were presented for selected 7, 5, and 3 coordinate axes-the ones that are the most essential entropy-wise (Table 2).

\subsection{Evaluating the methods of data grouping}

The end compatibility of image search results with their geological sources (verification of their compatibility with the actual rock type), presented in Table 3, suggest that the ineffectiveness level of the proposed methodology is ca. $17 \%$, for the minimum viable number of groups (Table 3 ). Still, comparing the results with regard to their visual similarity increases the reliability of the method. Depending on the number of groups, the visual similarity within a discovered cluster rises-from $89 \%$ for the minimum number of groups to almost $100 \%$ for over 50 groups. The Table 4 provides the statistics concerning the variability of correct visual matching. The evaluation was performed in a non-automatic way-the similarity of each searched

Table 3 The end compatibility of image search results with their geological sources, for different data grouping methods (\%)

\begin{tabular}{lllllllll}
\hline Number of groups & 19 & 30 & 40 & 50 & 60 & 70 & 80 & 90 \\
\hline$k$-means & 85.00 & 83.26 & 87.95 & 90.09 & 92.36 & 91.90 & 92.78 & 93.12 \\
$k$-medoids & 74.50 & 81.93 & 86.81 & 85.55 & 90.79 & 89.96 & 91.37 & 92.38 \\
MEC & 83.17 & 82.81 & 85.55 & 89.61 & 90.84 & 92.31 & 92.51 & 92.44 \\
\hline
\end{tabular}


Table 4 Evaluating the homogeneity of the visual similarity (\%) within one group, for the $k$-means grouping method

\begin{tabular}{|c|c|c|c|c|c|c|c|c|c|}
\hline Number of groups & 19 & 30 & 40 & 50 & 60 & 70 & 80 & 90 & 100 \\
\hline Arithmetic mean & 89.06 & 93.77 & 96.30 & 98.30 & 97.78 & 98.93 & 98.43 & 99.49 & 99.29 \\
\hline Median & 95.00 & 96.00 & 100.00 & 100.00 & 100.00 & 100.00 & 100.00 & 100.00 & 100.00 \\
\hline Mode & 100.00 & 100.00 & 100.00 & 100.00 & 100.00 & 100.00 & 100.00 & 100.00 & 100.00 \\
\hline Standard deviation & 13.12 & 7.32 & 8.43 & 5.25 & 5.59 & 5.17 & 6.51 & 3.55 & 4.76 \\
\hline Range & 42.00 & 22.00 & 40.00 & 30.00 & 31.00 & 30.00 & 40.00 & 32.00 & 43.00 \\
\hline Variance & 162.6 & 53.63 & 71.03 & 27.60 & 31.22 & 26.73 & 37.33 & 12.62 & 22.61 \\
\hline Coefficient of variation & 0.16 & 0.08 & 0.09 & 0.05 & 0.06 & 0.05 & 0.07 & 0.04 & 0.05 \\
\hline
\end{tabular}

group was determined by a researcher. Figure 6 shows that the rock structures, which are different geological objects, sometimes look alike. In such cases, the system-as it is supposed to-classifies them as one group of visually similar? images. This is why the matching results are slightly less accurate when it comes to group affiliation-hence, the noticeable differences in Tables 3 and 4 .

The $c$-means algorithm is for cluster analysis in which the allocation of data points to clusters is not binary (also called "all-or-nothing") but "fuzzy" what means that in the same sense as fuzzy logic every point has a degree of belonging to clusters, rather than belonging completely to just one cluster. The problem here are the values which obtain the same probability of belonging - unfortunately, the human interpretation is required. Of the 19 selected groups, algorithm emerged five groups, which tend to be visually dominant. Examples of the types of rocks, which are classified in groups 2 and 7 are shown in Fig. 7. In the overall assessment, this method based on the presented parameters gives the results difficult to automate. It can be

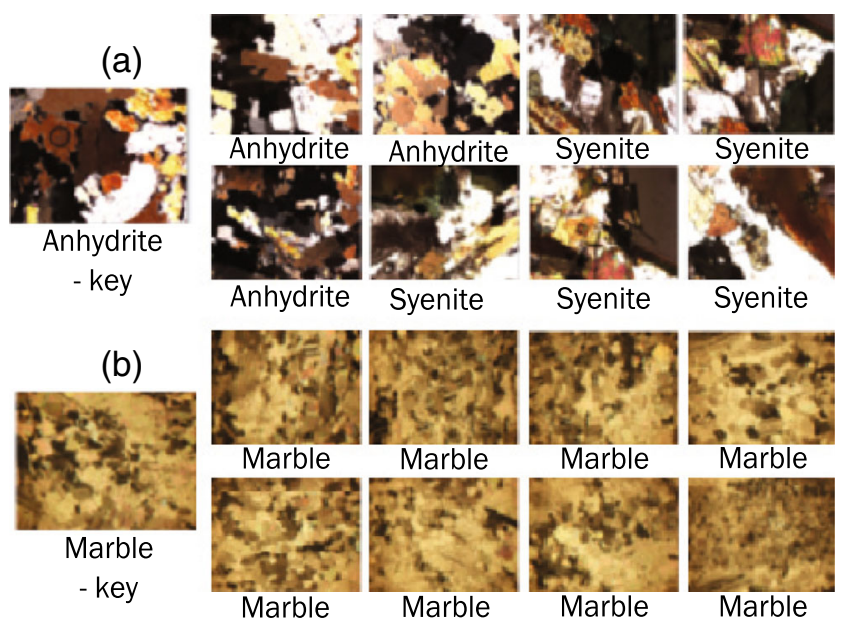

Fig. 6 The end results of the search of images, that are visually similar to the search key: anhydrite (results are incorrect from the geological perspective (a)) and marble (results are correct from the petrographic perspective $(\mathbf{b}))-k$-means algorithm used as an aid, but requires human intervention at a higher level than previously presented methods.

\subsection{Evaluating the method of data classification}

Table 5 shows the results of the method based on the analysis of $k$-nearest neighbors for $k=(20,100)$, changing with the 20th step, were presented. The obtained classification results were very good. The accuracy of the classification depends on the number of similar images being searched for. It can be assumed that, for $k$ smaller than 100-nearest neighbors, the accuracy is close to $100 \%$, in most cases. The higher the parameter $k$, the less accurate the recognizability. Figures 8, 9, and 10 present the most accurately matched classification results. The classification was performed by means of the $k$-nearest neighbors method, where $k=9$. Three examples of rock structures were chosen: the dolomite I (Fig. 8)-perfect matching; the sandstone III (Fig. 10)—petrographic compatibility ca. $80 \%$, visual compatibility $100 \%$; and the sandstone II (Fig. 9)—geological and visual compatibility ca. $60 \%$. The search key was an image-in each case, an element of the analyzed database.

(a)

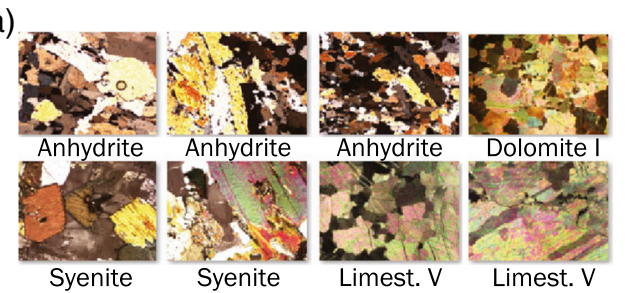

(b)

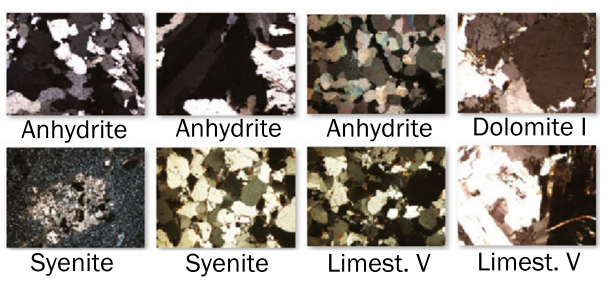

Fig. 7 Examples of the types of rocks, which are classified in groups 2 (a) and 7 (b) $-c$-means algorithm 
Table 5 The compatibility of image search results with their geological source for the kNN classification method—geological similarity $(g)$, visual similarity $(v)(\%)$

\begin{tabular}{|c|c|c|c|c|c|c|c|c|c|c|c|}
\hline & Number of neighbors & 20 & & 40 & & 60 & & 80 & & 100 & \\
\hline ID & Type of similarity & $g$ & $v$ & $g$ & $v$ & $g$ & $v$ & $g$ & $v$ & $g$ & $v$ \\
\hline 1 & Dolomite I & 100.0 & 100.0 & 100.0 & 100.0 & 100.0 & 100.0 & 100.0 & 100.0 & 100.0 & 100.0 \\
\hline 2 & Dolomite II & 100.0 & 100.0 & 100.0 & 100.0 & 100.0 & 100.0 & 100.0 & 100.0 & 100.0 & 100.0 \\
\hline 3 & Dolomite III & 100.0 & 100.0 & 100.0 & 100.0 & 100.0 & 100.0 & 100.0 & 100.0 & 100.0 & 100.0 \\
\hline 4 & Sandstone I & 100.0 & 100.0 & 100.0 & 100.0 & 100.0 & 100.0 & 100.0 & 100.0 & 100.0 & 100.0 \\
\hline 5 & Sandstone II & 60.0 & 60.0 & 50.0 & 50.0 & 53.5 & 53.5 & 47.5 & 50.0 & 52.0 & 60.0 \\
\hline 6 & Sandstone III & 80.0 & 100.0 & 82.5 & 100.0 & 76.7 & 100.0 & 80.0 & 100.0 & 82.0 & 100.0 \\
\hline 7 & Granite & 100.0 & 100.0 & 95.0 & 95.0 & 81.7 & 81.7 & 61.3 & 100.0 & 65.0 & 65.0 \\
\hline 8 & Quartzite & 100.0 & 100.0 & 100.0 & 100.0 & 100.0 & 100.0 & 100.0 & 100.0 & 100.0 & 100.0 \\
\hline 9 & Marble & 100.0 & 100.0 & 100.0 & 100.0 & 90.0 & 100.0 & 88.8 & 100.0 & 80.0 & 100.0 \\
\hline 10 & Slate crystalline I & 100.0 & 100.0 & 100.0 & 100.0 & 100.0 & 100.0 & 100.0 & 100.0 & 100.0 & 100.0 \\
\hline 11 & Slate crystalline II & 100.0 & 100.0 & 97.5 & 100.0 & 98.3 & 100.0 & 98.8 & 100.0 & 96.0 & 97.0 \\
\hline 12 & Porphyry & 100.0 & 100.0 & 100.0 & 100.0 & 100.0 & 100.0 & 100.0 & 100.0 & 100.0 & 100.0 \\
\hline 14 & Limestone I & 100.0 & 100.0 & 100.0 & 100.0 & 100.0 & 100.0 & 100.0 & 100.0 & 97.0 & 97.0 \\
\hline 15 & Limestone II & 100.0 & 100.0 & 100.0 & 100.0 & 100.0 & 100.0 & 100.0 & 100.0 & 100.0 & 100.0 \\
\hline 13 & Limestone III & 100.0 & 100.0 & 100.0 & 100.0 & 100.0 & 100.0 & 100.0 & 100.0 & 99.0 & 100.0 \\
\hline 17 & Limestone IV & 100.0 & 100.0 & 100.0 & 100.0 & 100.0 & 100.0 & 100.0 & 100.0 & 100.0 & 100.0 \\
\hline 16 & Limestone V & 100.0 & 100.0 & 67.5 & 80.0 & 53.3 & 80.0 & 50.0 & 67.5 & 40.0 & 60.0 \\
\hline 18 & Syenite & 100.0 & 100.0 & 92.5 & 100.0 & 93.3 & 100.0 & 91.3 & 100.0 & 89.0 & 100.0 \\
\hline 19 & Anhydrite & 100.0 & 100.0 & 77.5 & 100.0 & 60.0 & 100.0 & 53.8 & 100.0 & 57.0 & 95.0 \\
\hline
\end{tabular}

It was classified as the nearest one within the analyzed space of features, which confirms the accuracy of the algorithm.

\section{Recapitulation}

In the paper, it was demonstrated that both the grouping methods and the non-model data classification methods can be treated as effective mathematical methods in the process of searching for visually similar rock structures by image. The proposed concept of the system-based on a

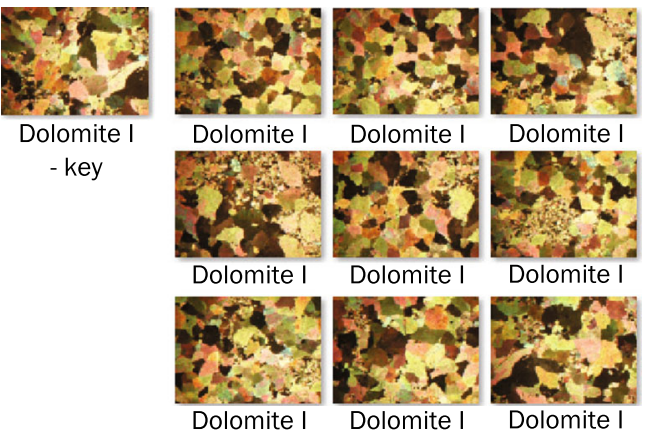

Fig. 8 The search results of $k=9$ nearest neighbors for the selected key the dolomite I (ID 1, Table 5) space of features defined by the authors-matches images to a selected visual model very effectively. As the rocks did not reveal visual homogeneity within one particular type, two types of results were presented: those concerning the geological origin and those concerning visual similarity.

Searching for visually similar images yielded the accuracy of ca. $90 \%$ for the minimum viable number of groups, to almost $100 \%$ for relatively large partitioning into groups (ca. 100). The petrographic matching of these rocks was from 80 to $95 \%$ for selected mathematical methods. A considerable drawback, as far as the grouping methods are concerned, is the fact that-although the methodology in
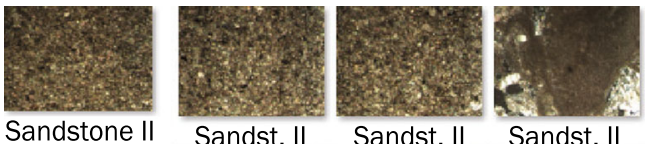

- key

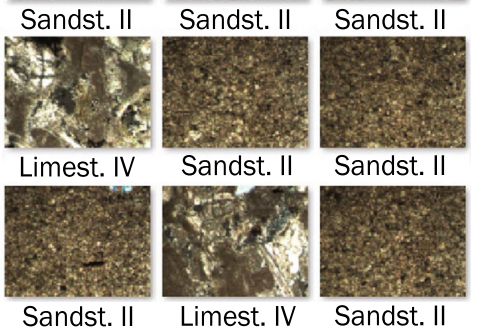

Fig. 9 The search results of $k=9$ nearest neighbors for the selected key the sandstone II (ID 5, Table 5) 


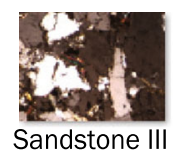

- key

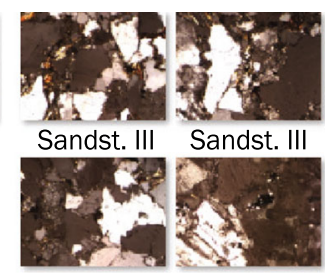

Sandst. III

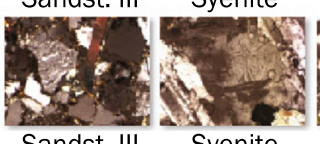

Sandst. III Syenite

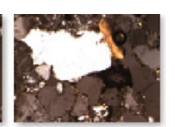

Sandst. III
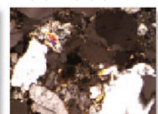

Sandst. III

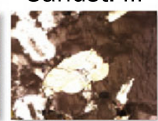

Syenite
Fig. 10 The search results of $k=9$ nearest neighbors for the selected key the sandstone III (ID 6, Table 5)

question is very effective when it comes to finding consistent clusters of similar images - the user cannot be sure if the obtained group of results encompasses all the similar images. In such a situation, the method of $k$-nearest neighbors proved to be of help. It guarantees that the $n$ number of the requested images is the closest to the model in the previously defined space of features (i.e., the images are most similar). More often than not, the visual matching happens to be $100 \%$ (for 100 most similar images), and the compatibility with the petrographic type of the rock is between 60 and $100 \%$.

The aim of the proposed methodology is to facilitate the decision-making process involved in searching for images that are similar to a given model. In the opinion of the authors of the paper, it satisfies the necessary criteria. Thus, it can be applied to the issue of finding images in databases and searching through discs in order to find images of visually similar rock structures, as well as going over video sequences in order to locate frames presenting such structures.

Acknowledgments This work was financed by the AGH - University of Science and Technology, Faculty of Geology, Geophysics and Environmental Protection as a part of statutory project.

Open Access This article is distributed under the terms of the Creative Commons Attribution License which permits any use, distribution, and reproduction in any medium, provided the original author(s) and the source are credited.

\section{References}

1. Aigrain, P., Zhang, H., Petkovic, D.: Content-based representation and retrieval of visual media: a state-of-the-art review. Multimed. Tools Appl. 3(3), 179-202 (1996)

2. Bach, J.R., Fuller, C.E., Gupta, A., Horowitz, B., Jain, R., Shu, C.F.: U.S. Patent no. 5,893,095, DC: U.S. Patent and Trademark Office, Washington (1999)

3. Baykan, N.A., Ylmaz, N.: Mineral identification using color spaces and artificial neural networks. Comput. Geosci. 36(1), 9197 (2010)
4. Cannon, R.L., Dave, J.V., Bezdek, J.C.: Efficient implementation of the fuzzy c-means clustering algorithms, pattern analysis and machine intelligence. IEEE Trans. 2, 248-255 (1986)

5. Celeux, G., Soromenho, G.: An entropy criterion for assessing the number of clusters in a mixture model. J. Classif. 13(2), 195-212 (1996)

6. Dudani, S.A.: The distance-weighted k-nearest-neighbor rule. Systems, man and cybernetics. IEEE Trans (4), 325-327 (1976)

7. Grunbock, C., King, M.T., Mannby, C.F., Smith, M.J.: U.S. Patent Application 12(964), 662 (2010)

8. Gudivada, V.N., Raghavan, V.V.: Content based image retrieval systems. Computer 28(9), 18-22 (1995)

9. Haneberg, W.C.: Simulation of 3D block populations to characterize outcrop sampling bias in bimrocks, Felsbau Rock and Soil Engineering. J. Eng. Geol. Geomech. Tunn. 22(5) (2004)

10. Hardy, A.: On the number of clusters. Comput. Stat. \& Data Anal. 23(1), 83-96 (1996)

11. Herbin, M., Bonnet, N., Vautrot, P.: Estimation of the number of clusters and influence zones. Pattern Recogn. Lett. 22(14), 15571568 (2001)

12. Holden, E.J., Moss, S., Russell, J.K., Dentith, M.C.: An image analysis method to determine crystal size distributions of olivine in kimberlite. Comput. Geosci. 13(3), 255-268 (2009)

13. Jain, A.K., Vailaya, A.: Image retrieval using color and shape. Pattern Recogn. 29(8), 1233-1244 (1996)

14. Kasvand, T.: Computerized vision for the geologist. J. Int. Assoc. Math. Geol. 15(1), 3-23 (1983)

15. Li, X.R., Zhang, K., Jiang, T.: Minimum entropy clustering and applications to gene expression analysis. In: Computational Systems Bioinformatics Conference, vol. 2004, pp. 142-151 (2004)

16. Marmo, R., Amodio, S., Tagliaferri, R., Ferreri, V., Longo, G.: Textural identification of carbonate rocks by image processing and neural network: Methodology proposal and examples. Comput. Geosci. 31(5), 649-659 (2005)

17. Marques, O., Furht, B.: Content-based visual information retrieval. Distrib. Multimed. Databases: Tech. Appl., 37-57 (2001)

18. Meng, H.D., Song, Y.C., Song, F.Y., Shen, H.T.: Research and application of cluster and association analysis in geochemical data processing. Comput. Geosci. 15(1), 87-98 (2011)

19. Mlynarczuk, M., Gorszczyk, A., Slipek, B.: The application of pattern recognition in the automatic classification of microscopic rock images. Comput. Geosci. 60, 126-133 (2013)

20. Mlynarczuk, M.: Description and classification of rock surfaces by means of laser profilometry and mathematical morphology. Int. J. Rock Mech. Min. Sci. 47(1), 138-149 (2010)

21. Moore, B.: Principal component analysis in linear systems: controllability, observability, and model reduction, automatic control. IEEE Trans. 26(1), 17-32 (1981)

22. Obara, B., Kozusnikova, A.: Utilisation of the image analysis method for the detection of the morphological anisotropy of calcite grains in marble. Comput. Geosci. 11(4), 275-281 (2007)

23. Park, H.S., Jun, C.H.: A simple and fast algorithm for k-medoids clustering. Expert Syst. Appl. 36(2), 3336-3341 (2009)

24. Porter, M.L., Wildenschild, D.: Image analysis algorithms for estimating porous media multiphase flow variables from computed microtomography data: a validation study. Comput. Geosci. 14(1), 15-30 (2010)

25. Singh, N., Singh, T.N., Tiwary, A., Sarkar, K.M.: Textural identification of basaltic rock mass using image processing and neural network. Comput. Geosci. 14(2), 301-310 (2010)

26. Smeulders, A.W., Worring, M., Santini, S., Gupta, A., Jain, R.: Content-based image retrieval at the end of the early years. Pattern Analysis and Machine Intelligence. IEEE Trans. 22(12), 1349$1380(2000)$ 
27. Thompson, S., Fueten, F., Bockus, D.: Mineral identification using artificial neural networks and the rotating polarizer stage. Comput. Geosci. 27(9), 1081-1089 (2001)

28. Tadeusiewicz, R., Ogiela, M.R.: Medical image understanding technology, artificial intelligence and soft computing for image understanding. Springer-Verlag, Berlin, Heildelberg (2004)

29. Kurgan, L.A., Cios, K.J., Tadeusiewicz, R., Ogiela, M., Goodenday, L.S.: Knowledge discovery approach to automated cardiac SPECT diagnosis. Artif. Intell. Med. 23(2), 149-169 (2001)

30. Wagstaff, K., Cardie, C., Rogers, S., Schrdl, S.: Constrained k-means clustering with background knowledge. ICML 1, 577584 (2001)

31. Weinberger, K.Q., Blitzer, J., Saul, L.K.: Distance metric learning for large margin nearest neighbour classification. In: Advances in neural information processing systems, pp. 1473-1480 (2005) 